\title{
A liquid biopsy-based approach identifies myeloid cells, STAT3 and arginase- 1 as predictors of glioma risk score and patients' survival
}

\section{Paola Del Bianco}

IOV IRCCS: Istituto Oncologico Veneto Istituto di Ricovero e Cura a Carattere Scientifico

\section{Laura Pinton}

IOV IRCCS: Istituto Oncologico Veneto Istituto di Ricovero e Cura a Carattere Scientifico

\section{Sara Magri}

University of Padova Department of Surgical Oncology and Gastroenterology: Universita degli Studi di

Padova Dipartimento di Scienze Chirurgiche Oncologiche e Gastroenterologiche

\section{Stefania Canè}

University of Verona: Universita degli Studi di Verona

\section{Elena Masetto}

IOV IRCCS: Istituto Oncologico Veneto Istituto di Ricovero e Cura a Carattere Scientifico

\section{Daniela Basso}

University of Padova Department of Medicine: Universita degli Studi di Padova Dipartimento di

Medicina

\section{Marta Padovan}

IOV IRCCS: Istituto Oncologico Veneto Istituto di Ricovero e Cura a Carattere Scientifico

\section{Francesco Volpin}

Azienda Ospedale Università Padova: Azienda Ospedale Universita Padova

\section{Domenico D'Avella}

Azienda Ospedale Università Padova: Azienda Ospedale Universita Padova

\section{Giuseppe Lombardi}

IOV IRCCS: Istituto Oncologico Veneto Istituto di Ricovero e Cura a Carattere Scientifico

\section{Vittorina Zagonel}

IOV IRCCS: Istituto Oncologico Veneto Istituto di Ricovero e Cura a Carattere Scientifico

\section{Vincenzo Bronte}

University of Verona: Universita degli Studi di Verona

\section{Alessandro Della Puppa}

Università degli Studi di Firenze: Universita degli Studi di Firenze

SUSANNA MANDRUZZATO ( $\sim$ susanna.mandruzzato@unipd.it)

University of Padua https://orcid.org/0000-0002-0707-995X 
Research

Keywords: Glioma, Immune evasion, myeloid-derived suppressor cells, biomarkers, STAT3, ARG1

Posted Date: September 22nd, 2021

DOI: https://doi.org/10.21203/rs.3.rs-895672/v1

License: (c) (i) This work is licensed under a Creative Commons Attribution 4.0 International License. Read Full License 


\section{Abstract \\ Background}

Although gliomas are strictly confined to the central nervous system, their negative influence over the immune system can extend to peripheral circulation. The immune suppression exerted by myeloid cells is capable of affecting both response to therapy and disease outcome. Here we analyzed the expansion of several myeloid parameters in the blood of low- and high-grade gliomas and assessed their relevance as biomarkers of disease and clinical outcome.

\section{Methods}

Peripheral blood was obtained from 134 low- and high-grade glioma patients before surgery and treatment. Myeloid cell subsets such as total CD $14^{+}, \mathrm{CD} 14^{+} / \mathrm{p}-\mathrm{STAT} 3^{+}, \mathrm{CD} 14^{+} / \mathrm{PD}-\mathrm{L} 1^{+}, \mathrm{CD} 15^{+}$cells and 4 myeloid derived suppressor cell (MDSC) subsets, were evaluated by multiparametric flow cytometry. Arginase-1 (ARG1) quantity and activity was determined in the plasma. Principal component analysis was performed to define correlations between myeloid markers. Multivariable logistic regression model was used to obtain a diagnostic score to discriminate glioma patients from healthy controls, and between each glioma grade. A glioblastoma prognostic model was determined by multiple Cox regression using clinical and myeloid parameters.

\section{Results}

In the blood of glioma patients, changes in myeloid parameters associated with immune suppression were identified and allowed us to define a diagnostic score calculating the risk of being a glioma patient, that included $\mathrm{CD}_{15}{ }^{+}$cells, MDSC1, MDSC3, p-STAT3 and ARG1 activity. Of note, the same parameters, together with age, can also be used to calculate the risk score in differentiating each glioma grade. Finally, a prognostic model for glioblastoma patients stemmed out from a Cox multiple analysis, highlighting the role of MDSC, p-STAT3 and ARG1 activity together with clinical parameters in predicting the patient outcome.

\section{Conclusions}

This work emphasizes the role of systemic immune suppression carried out by myeloid cells in gliomas. The identification of biomarkers associated with immune landscape, diagnosis and outcome of glioblastoma patients lays the ground for their clinical use for stratification and follow up.

\section{Background}


Gliomas represent a heterogeneous group of primary brain tumors. Although many advances have been obtained at the molecular level contributing to patient's stratification, these findings did not translate into new drugs development or significant improvements in patient's treatment. Prediction of clinical outcome is mainly based on clinicopathological and genetic factors that do not evaluate accurately survival outcomes, and, moreover, genetic factors depend on surgical resection, or tumor biopsy (1). Prognosis is poor, especially for high-grade gliomas, due to aggressive invasiveness, high proliferation rate and resistance to standard treatments. The relationship between immune system and gliomas has also been studied, and it revealed a strong control of the tumor on the existing anti-tumor activity, as gliomas are characterized by a profound local and systemic immune suppression (2). One of the mechanisms exploited by the tumors depends on the release of soluble factors that drive the generation and recruitment of altered myeloid cells displaying a potent immune suppressive activity (3). Indeed, during tumor progression myelopoiesis is diverted from its normal pathway and often results in the expansion and accumulation of myeloid-derived suppressor cells (MDSCs), a heterogeneous population frequently expanded in different types of cancer, impairing antitumor innate and adaptive immune responses $(4,5)$. The phenotype of MDSCs shows a complex plasticity that depends upon the particular combination of tumor-derived soluble factors that are present in the tumor microenvironment. In humans, three main subsets can be distinguished: polymorphonuclear MDSCs (PMN-MDSCs), monocytic MDSCs (M-MDSCs), and early-stage MDSCs (e-MDSCs) $(5,6)$, although each contains more than one cell population $(7,8)$. In line with their function of suppressing the immune response, MDSC levels correlate proportionally with tumor burden (9), and are associated with tumor progression and lack of response to therapy $(10,11)$. Concerning glioblastoma (glioma grade IV, GBM), previous works reported the expansion of subsets of MDSCs (12-16), and an association of levels of $\mathrm{CD} 11^{+} / \mathrm{CD}_{3} 3^{+} / \mathrm{HLA}^{-D R^{\text {low }} /-}$ MDSCs with survival (17). Interestingly, based on these observations a phase 0/I dose-escalation trial has been conducted in recurring patients with metronomic capecitabine, to reduce MDSC levels (18).

Among the several mechanisms of the immune suppressive machinery that have been described in MDSCs, it has been reported the expression of programmed death-ligand 1 (PD-L1), the activation of Signal Transducer and Activator of Transcription 3 (STAT-3), and of the enzyme arginase-1 (ARG1) (1921). In fact, another mechanism by which immune cells promote cancer growth is the depletion of essential nutrients that are required by lymphocytes, like arginine. Reduction of arginine levels can be obtained by ARG1, resulting in antigen-activated T cells proliferation arrest (22). High levels of arginase have been reported in several cancer types, thus providing an attractive target for anticancer immunotherapy (23). A few studies documents the role of ARG1 (12, 24), and PD-L1 (25) in the immune suppression in GBM, but data are scarce in circulating myeloid cells from low grade gliomas (12).

Recently, suppressive M-MDSCs from pancreatic ductal carcinoma patients were characterized as $\mathrm{STAT}^{+} / \mathrm{ARG}^{+} / \mathrm{CD} 14^{+}$cells with a distinct gene signature in which STAT3 has a main role in driving MDSC function (20). Once activated, this pathway inhibits T-cell proliferation and reduces $T$ cell effector functions. The presence of an expansion of MDSCs can be considered not only as a hallmark of immune 
suppression, but also as a biomarker of disease, or disease progression. Based on these premises, levels of circulating MDSCs have been regarded as a tool to monitor disease progression (26).

Biomarkers for patient stratification and for response to therapy are an important tool in oncology. In brain tumors the possibility of using body fluids as a source of biomarkers to diagnose and define disease progression is attractive since it is minimally invasive, thus circumventing the need of intracranial sampling. For this reason, circulating tumor cells, exosomes, proteins, nucleic acids and metabolites have been proposed as potential biomarkers in gliomas, but, at present, none is yet in clinical use (27).

In this prospective study we analyzed in the blood of $(\mathrm{HD})$ and of low and high-grade glioma patients a number of soluble and cell-associated markers of myeloid cells to identify new biomarkers capable of predicting diagnosis and clinical outcome. The analysis of these myeloid parameters allowed to develop a diagnostic score that classified HDs versus glioma patients, and versus grade II, III and GBM patients. Finally, a GBM prognostic risk model was identified that combines clinical and myeloid parameters.

\section{Methods}

\section{Patients and samples}

Patients were prospectively recruited at the Department of Neurosurgery, Padova University Hospital, Italy, from 2016 to 2019, then followed at the Veneto Institute of Oncology, Padova, Italy. Patients were eligible if they were older than 18 years with histologically confirmed diagnosis of glioma. Clinical data and molecular analyses such as MGMT methylation status and IDH mutational status were also collected, prospectively. A total of 134 treatment-naive glioma patients ( $\mathrm{n}=19$ grade $\mathrm{Il}, \mathrm{n}=14$ grade $\mathrm{II}, \mathrm{n}=101$ grade IV, Table 1) undergoing surgery, and 65 healthy donors (HDs), matched for age and sex, as control, were included in this study. Of these, 140 (104 patients and $36 \mathrm{HDs}$ ) were analyzed for myeloid cell-associated markers (CD14 ${ }^{+}, \mathrm{CD} 15^{+}, \mathrm{MDSC} 1, \mathrm{MDSC} 2, \mathrm{MDSC} 3, \mathrm{MDSC} 4$; cohort 1), 86 (63 patients and $23 \mathrm{HDs}$ ) were analyzed for STAT3 and PD-L1 (cohort 2), and 82 (64 patients and 18 HDs) for ARG1 quantity and activity (cohort 3), as shown in Table S1. Cohorts 1 and 2 shared 4 HDs and 15 patients; cohorts 1 and 3 shared 8 HDs and 30 patients; twenty-six patients were included in all 3 cohorts. Details of subject inclusion are reported in Figure 1. Only subjects with complete data were eligible for the analyses. The study protocol was reviewed and approved by the ethical committee of the IOV-IRCCS and of Padova University Hospital and all patients gave their informed consent.

\section{Flow cytometry}

Peripheral blood was obtained from patients at surgery before anesthesia induction, or the day before surgery, and immediately processed. Briefly, peripheral blood mononuclear cells (PBMCs) were isolated by density gradient centrifugation on Ficoll-Paque PLUS (GE Healthcare-Amersham, NJ, USA), followed by dextran sedimentation to obtain PMNs, as previously described (28). Fresh unrefrigerated whole blood from patients and from HD was stained with monoclonal antibodies to analyze myeloid cell subsets, within two hours after withdraw. MDSC subsets were identified by a 7-color staining, containing anti- 
CD11b Alexa700 (BD Biosciences), anti-CD14 APC-H7 (BD Biosciences), anti-CD15 V450 (BD

Biosciences), anti-CD33 PE-Cy7 (eBioscience), anti-IL4Ra PE (R\&D SYSTEMS), Lineage cocktail (Lin) FITC (BD Biosciences), anti-HLA-DR APC (BD Biosciences) and the immunophenotyping was standardized as described below. An Ab mixture containing anti-CD274 (PD-L1) PE (eBioscience), anti-CD14 APC-H7 (BD Biosciences), anti-HLA-DR APC (BD Biosciences) was used to analyze the expression of PD-L1 in myeloid cell populations.

To analyze the intracellular expression of ARG1, PBMCs and PMNs were isolated from the peripheral blood of HDs and glioma patients as previously described (28), stained with anti-CD14 FITC (BD Biosciences) and anti-CD15 V450 (BD Biosciences), then cells were fixed and permeabilized with Cytofix/Cytoperm (BD Biosciences) for 20 minutes at $4^{\circ} \mathrm{C}$. Then PBMCs and PMNs were stained for 30 minutes at $4^{\circ} \mathrm{C}$ with mouse monoclonal antibody anti-human ARG1 (clone 1.10, (10)) conjugated to Alexa Fluor (AF)-647 by SAIVI ${ }^{\mathrm{TM}}$ Rapid Antibody Labeling Kits Thermo Fisher, following the manufacturer's instructions.

To detect intranuclear p-STAT3, PBMCs were stained with anti-CD14 FITC (BD Biosciences), then fixed with $1 \%$ formaldehyde for 10 minutes at $37^{\circ} \mathrm{C}$, washed twice with PBS $4 \%$ FBS and permeabilized with ice cold $96 \%$ methanol for 15 minutes at $-20^{\circ} \mathrm{C}$. After removing the methanol by washing twice with PBS $4 \%$ FBS, PBMCs were stained with anti-human p-STAT3 (Tyr705) mAb (Cell Signaling Technology, MA, USA) and then with donkey anti-rabbit AF-647 IgG antibody (BioLegend) for 30 minutes at room temperature (RT).

\section{Standardization of MDSCs staining acquisition and analysis}

To standardize MDSC subsets' evaluation, a dilution of antibodies that maximizes the signal to noise ratio was chosen based on single antibodies titration. In addition, a protocol to monitor the performance of antibodies against HLA-DR and IL4Ra was set-up. Briefly, an EBV-B cell line that constitutively expresses these markers at high expression intensity was used as reference, as previously described (7). To reduce inter-assay variance, the $B$ cell line was fixed and permeabilized in large batches and a single vial was run in parallel to blood staining for each patient. Acquisition of control cells was performed before the blood sample, to evaluate whether the mean fluorescence intensity (MFI) of HLA-DR or IL4Ra fell in the range of tolerance built by repeated staining of the control cells. In addition, for each patient, fluorescence minus one (FMO) for HLA-DR and IL4Ra were used as negative controls. To monitor the performance of the LSRII flow cytometer (BD Biosciences), the potential variation of the performance of the instrument was assessed, using a protocol after Perfetto et al. (29)

\section{Confocal microscopy}

PBMCs and PMNs were seeded on coverslips for 2 hours in 24-well plates and washed three times with PBS to eliminate non-adhering cells, fixed with 4\% paraformaldehyde (Sigma-Aldrich) for 10 min at RT, then blocked with PBS containing $0.05 \%$ Triton and $20 \%$ normal goat serum (NGS Vector Laboratories, Burlingame, CA, USA) for 2 hours at RT. Cells were stained for CD14 and ARG1 using an anti-CD14 Cy3 
(Bioss) and anti-human Arginase-1 clone 1.10 conjugated with AF-647, and incubated overnight at $4^{\circ} \mathrm{C}$. DNA was visualized with DAPI (Sigma-Aldrich). Samples were analyzed under a laser scanning confocal microscope (Leica TCS SP5, Wetzlar, Germany) equipped with 4 lasers (405nm/ Argon-458, 476, $488,494,514 \mathrm{~nm}-/ 561 \mathrm{~nm} / 633 \mathrm{~nm}$ ), and results were analyzed by Las X (Leica Microsystems).

\section{Determination of ARG1 levels and activity}

Plasma level of ARG1 was analyzed using Arginase Liver Type Human ELISA (BioVendor Laboratory Medicine Inc.) following the manufacturer's instructions. Plasma was obtained upon centrifugation over Ficoll-Paque Plus of peripheral blood. The supernatant was collected and further spun at $1300 \mathrm{rpm}$ at $4^{\circ} \mathrm{C}$ for 6 minutes and stored at $-80^{\circ} \mathrm{C}$. Samples were assayed in duplicates and ARG1 concentration was extrapolated from the standard curve. Arginase activity was tested in plasma samples by measuring the production of urea, at pH 7.2 and 9.5, as detailed in Supplementary Materials and Methods.

\section{Statistical analysis}

Continuous variables were summarized using median and interquartile range, categorical variables as frequencies and percentages. A two-tailed Mann-Whitney test, adjusted for sex and age, followed by the Benjamini-Hochberg multiple testing correction, was used to address the pairwise comparisons of each biomarker distribution between HDs and low- and high-grade glioma patients. The strength and direction of existing structures and patterns between the levels of all markers within each cohort was evaluated using a Pearson correlation analysis. A hierarchical clustering on principal components approach was used to identify groups of subjects sharing similarities. As preparatory steps to clustering, a principal component analysis (PCA) analysis was performed to remove the noise from the data to keep only relevant information and make the former more meaningful and stable. Only subjects with complete data were eligible for the analyses. Multiple logistic and multinomial logistic regression models were estimated to develop a diagnostic score using each biomarker categorized according to high and low levels. The selection of variables was based on Akaike's Information Criterion in order to reduce model complexity. Optimal cutpoints for each soluble and cell-associated marker of myeloid cells were selected using a criterion based on minimization of the most frequent error (30). The odds ratios (OR) were reported with their $95 \%$ confidence interval $(\mathrm{Cl})$. A repeated (3-repeats) 5 -fold cross-validation was utilized for internal validation. Accuracy, specificity, and sensitivity were calculated to assess the prediction error. Among grade IV glioma patients, clinical outcome was analyzed in terms of overall survival (OS), defined as the time from the date of surgery to death. Patients who did not develop an event during the study period were censored at the date of last observation. The median follow-up time was based on the reverse Kaplan-Meier estimator. The survival probabilities were estimated using the Kaplan-Meier method and median survival was reported with a $95 \% \mathrm{Cl}$ calculated according to Brookmeyer and Crowley. The association of clinical characteristics and markers with overall survival was investigated in multiple Cox proportional hazards regression models. No deviation from the proportional hazards assumption was found by the test statistic of Grambsch and Therneau. Clinical prognostic factors incorporated in the model include age at surgery, sex, ECOG performance status (ECOG PS) (0-1, 2-4), type of surgery (radical, 
other), whether patients received Stupp's treatment, MGMT (O[6]-methylguanine-DNA methyltransferase) promoter methylation (present, absent), IDH (Isocitrate dehydrogenase) status (mutated, wild type (WT)). Markers within each cohort were dichotomized with cut points corresponding to the most significant relation with the outcome, estimated by maximizing the discriminative ability of the Cox model. The best model was selected with the lower Akaike Information Criteria and the concordance index (C-index) was used to evaluate the discrimination of the model. Bootstraps with 1,000 resamples were calculated to correct the C-index.

All statistical tests were two-sided and a p value $<0.05$ was considered statistically significant. Statistical analyses were performed using the RStudio (RStudio: Integrated Development for R. RStudio Inc., Boston, MA, US).

\section{Results}

\section{Expansion of suppressive myeloid cell subsets in the peripheral blood of glioma patients}

To determine the role of circulating immune parameters related to immune suppression in the clinical outcome of glioma patients, we set out to perform a detailed immunophenotypical analysis of circulating myeloid cells by multicolor flow cytometry in 134 patients undergoing surgery for a suspect glioma. To this aim peripheral blood samples were prospectively collected from 3 cohorts of patients and control HDs, as detailed in Figure 1, and parameters analyzed in each of the 3 cohorts are indicated in Table S1. As previously reported by us and others (31-33), GBM patients had a significant increase in the percentage of $\mathrm{CD}_{14}{ }^{+}$circulating monocytes as compared to age and sex matched HDs, as well as a significant increase of PMNs, defined as $\mathrm{CD}_{15}{ }^{+}$cells (Fig. 2a). When we analyzed lower grade gliomas we observed that also these patients were characterized by a significant expansion of both monocytes and PMNs, with a significant increase going from lower grade to GBM (Fig. 2b). Although some of these glioma patients underwent preoperative dexamethasone and it is known that this treatment increases PMNs $(34,35)$, no clear data exist regarding its effect on monocytes (36). However, we did not find a significant difference in $\mathrm{CD} 14^{+}$cells from patients with or without preoperative dexamethasone (Supplementary Fig. S1). Collectively, these results indicate that the increase in CD14 ${ }^{+}$monocytes in glioma patients is not due to dexamethasone, and suggest a dysregulation of the myeloid compartment. In the same blood samples, we also evaluated MDSCs levels. Previous studies analyzed MDSCs in glioma patients, but few of them analyzed more than one MDSC subset at a time, and most studies evaluated MDSCs among PBMCs, thus excluding the potential presence of PMN-MDSC subsets present in whole blood, discarded after a ficoll gradient. In addition, also cryopreservation is well known to influence the evaluation of PMN-MDSC. Based on these considerations, we decided to use a standardized 7-color panel, stemming from our previous experience $(7,8,37)$, to detect in fresh whole blood the simultaneous presence of the following subsets: M-MDSC1 (CD14 $\left./ 4^{+} 4 \mathrm{Ra}^{+}\right), \mathrm{PMN}-\mathrm{MDSC} 2$

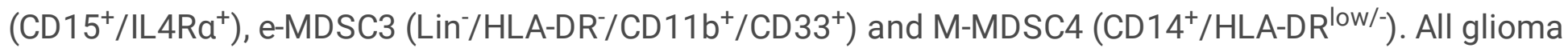
patients had a significant expansion of circulating MDSC1, 2 and 4 subsets in comparison to age- 
matched HDs (Fig. 2 panels c, $d$ and f). On the contrary, MDSC3 levels were significantly decreased in glioma grade II and IV patients with respect to HDs and in GBM as compared to grade III (Fig. 2e). Interestingly, MDSC4 were significantly increased in GBM compared to grade III gliomas, raising the possibility to use this marker in longitudinal studies to monitor evolution of grade III gliomas into GBM (Fig. 2f), since it is well known that GIII gliomas eventually evolve to GBM.

From these results, it appears that detection of circulating myeloid cells might represent a new tool for the follow-up of glioma patients, and that an altered myelopoiesis is associated with glioma grading progression.

\section{Activation of STAT3/PD-L1 axis in circulating monocytes from glioma patients}

We next investigated in circulating monocytes the activation of STAT3, one of the key players regulating tolerogenic activities of tumor-associated myeloid cells (21), by analyzing p-STAT3 expression in CD14 ${ }^{+}$ cells and found that its intensity significantly increased in all glioma grade compared to HD (Fig. $2 \mathrm{~g}$ ), thus suggesting an active involvement of this transcription factor in the modulation of immune suppression.

Once phosphorylated STAT3 moves to the nucleus, where it can induce the expression of PD-L1 by binding to its promoter and activating its transcription (38). Thus, to further analyze the regulation carried out by STAT3, we evaluated the expression of PD-L1 on circulating monocytes, and found that the percentage of monocytes expressing PD-L1 was significantly increased on grade III and IV gliomas, but not in grade II (Fig. 2h).

All together these observations indicate that increased glioma grading is associated with a rise in CD14 ${ }^{+}$ cells expressing activated STAT3 and PD-L1, suggesting their potential use as blood biomarkers.

\section{ARG1 activity increases with tumor stage}

It has been clearly demonstrated that ARG1, an enzyme constitutively expressed in PMNs and stored within intracellular granules, is a downstream target of activated STAT3, and that this regulation is also present in circulating MDSCs from cancer patients, in which it controls the immune suppressive activity (21). We thus investigated the presence of ARG1 in circulating PBMCs from glioma patients, by using confocal microscopy (Fig. 3a) and flow cytometry analysis (Fig. 3b). With both techniques, we observed the presence of a fraction of CD14 ${ }^{+}$monocytes expressing ARG1 (18.8\% by flow cytometry analysis and $21.4 \%$ by confocal microscopy evaluation) localized in the cytoplasm of the cells.

Previously, elevated circulating levels of ARG1 in GBM patients have been associated with PMN degranulation and immunosuppression (24). In addition, we recently demonstrated that high serum levels of ARG1 in pancreatic ductal adenocarcinoma patients were associated with high ARG1 activity (20). We thus measured ARG1 levels in the plasma samples obtained from 64 glioma patients ( $15 \mathrm{GIl}, 10 \mathrm{GIII}$ and $39 \mathrm{GBM}$ ) and found that ARG1 levels in glioma patients were significantly higher compared to HD control 
(Fig. 3c). We then assessed ARG1 enzymatic activity at both pH 7.2 and pH 9.5 (Fig. 3d and 3e). In both conditions, serum from glioma patients showed a significant increase in ARG1 activity that peaked in grade IV gliomas. Interestingly, ARG1 activity at pH 7.2 (determined as urea measured at pH 7.2) positively correlated with tumor grade, increasing from a median activity of $29.6 \mathrm{mg} / \mathrm{hour}$ of urea in HD to $85.4 \mathrm{mg}$ /hour in GII, 93.9 and $152.8 \mathrm{mg} /$ hour in GIII and GBM, arguing for ARG1 activity as a potential marker of glioma progression from grade III to GBM, although longitudinal studies are required to confirm and strengthen this conclusion.

\section{Correlation between immune suppressive markers}

To define the association of the different circulating parameters, we performed a PCA (Fig. 4) on the three groups of markers previously determined. When myeloid parameters considered in cohort 1 were evaluated, all variables displayed a moderate to strong positive correlation ( $r$ Pearson from 0.27 to 0.72 ), with the exception of MDSC3 that was negatively correlated with all the other variables ( $r$ Pearson from -0.26 to -0.42 ). Activation of STAT3 and PD-L1 expression were not correlated ( $r$ Pearson $=-0.09$ ) each other, while ARG1 activity showed a positive moderate correlation at the two different $\mathrm{pH}$ values $(r$ Pearson $=0.45$ ), but its quantity was not correlated from the activity both at $\mathrm{pH} 7.2$ and $\mathrm{pH} 9.5$ ( $\mathrm{r}$ Pearson $=0.14)$.

The hierarchical clustering on PCA, used to identify groups of markers with similarities, divided the subjects of cohort 1 into three main clusters, with one of them containing 35 out of 36 HDs (cluster 1 ), while glioma patients were divided in 2 groups (Cluster 2 and 3) (Fig. 4 upper panel). Cluster 1 was characterized by lower levels of CD14 ${ }^{+}, \mathrm{CD} 15^{+}$MDSC1, 2 and 4 and higher levels of MDSC3, in line with values obtained in HDs and, conversely, the opposite distinguished cluster 3 in which 32 out of $75 \mathrm{GBM}$ were grouped (Fig. 4, upper panel). The proposed clustering classified HD and the glioma patients with a sensitivity of $79.8 \%$, specificity of $97.2 \%$ and accuracy of $84.3 \%$, with 21 false negatives and 1 false positive, suggesting that the model can be useful for ruling in glioma patients.

As far as it concerns the markers expressed by suppressive monocytes such as PD-L1 and activated STAT3 reported in Fig. $2 \mathrm{~g}$-h, in a cohort of 63 glioma patients and 23 HD, two clusters could be identified. The first group comprised $87 \%$ of HD, and was characterized by lower percentage and intensity of activated STAT3, while higher levels were observed in group 2, in which expression of PD-L1 among monocytes was similarly expressed throughout the clustering (Fig. 4, central panel). This categorization classified HD and glioma patients with a sensitivity of $100 \%$, specificity of $0 \%$ and accuracy of $73.3 \%$.

Finally, the parameters related to ARG1 quantity and activity gave rise to 3 clusters (Fig. 4, lower panel). Cluster 1 was characterized by the lowest levels and activity of ARG1, cluster 2 had the samples with the highest quantity of ARG1, and an intermediate activity; cluster 3 contained samples with a low level of enzyme, but endowed with the highest activity. This clustering classified the HD and the glioma patients with a sensitivity of $100 \%$, specificity of $0 \%$ and accuracy of $78.1 \%$. Interestingly, all HDs fall in group 1 , while gliomas distributed in the 3 clusters, but the majority of GBM fell in group 3, and low-grade gliomas 
were mainly present in cluster 1 . It thus appears that the amount and activity of this enzyme characterize disease stage, and it has a significantly higher activity in high-grade gliomas.

\section{Development of a diagnostic score to identify biomarkers associated with disease and with disease stage.}

In order to define a diagnostic score, each biomarker was categorized according to high and low levels, and the association with the disease was tested first by univariate analysis (Table S2 and S3), followed by multiple logistic regression models considering in the stepwise model selection all markers within each cohort, together with age and sex. Analysis was performed including glioma patients as a single group, in a case control study (univariate in Table S2 and multivariate in Table 2a), or divided on the basis of pathological stage, from grade II to GBM (univariate in Table S3 and multivariate in Table 2b). When cell-associated myeloid markers were considered as biomarkers to differentiate HD from glioma patients, levels of $\mathrm{CD}_{15}{ }^{+}$cells, MDSC1, and MDSC3 emerged as independent factors predicting the presence of disease (Table 2a), with an overall accuracy of $87.1 \%$. In detail, high levels of CD $15^{+}$cells and MDSC1 were significantly associated with a high risk of disease (adjusted OR: 7.2, 95\% Cl: 2.2-24.1 and adjusted OR: $40.1,95 \% \mathrm{Cl}$ : 10.1-160.4, respectively), while high levels of MDSC3 showed a significantly lower probability to develop disease (adjusted OR: $0.1,95 \% \mathrm{Cl}$ : $0.02-0.4$ ). The logit transformation of the probability of glioma (any grade) risk was calculated as follows:

\section{$\operatorname{logit}(p)=-1.372+1.976 \times C D 15^{+}+3.691 \times M D S C 1-2.413 \times M D S C 3$}

Of note, when glioma patients were classified according to their grade, $C D 15^{+}$cells, MDSC1, and MDSC3 remained independent significantly predictors of GBM, and MDSC1 was the biomarker significantly associated to all grades of disease (Table $2 \mathrm{~b}$, accuracy of $72.9 \%$ ). In this case the formula for the risk score was:

$$
\begin{aligned}
& \operatorname{logit}\left(p_{G B M}\right)=-3.953+0.011 \times \text { age }+3.484 \times C D 15^{+}+4.175 \times M D S C 1-2.630 \times M D S C 3 \\
& \operatorname{logit}\left(p_{G I I}\right)=3.201-0.104 \times \text { age }+1.018 \times C D 15^{+}+3.362 \times M D S C 1-2.749 \times M D S C 3 \\
& \operatorname{logit}\left(p_{G I I I}\right)=0.208-0.052 \times \text { age }+1.066 \times C D 15^{+}+3.333 \times M D S C 1-1.651 \times M D S C 3
\end{aligned}
$$

Regarding PD-L1 and p-STAT3 expression, only the shift of intensity of expression of activated STAT-3 in monocytes (p-STAT3) remained an independent factor predicting the presence of disease (Table 2a, cohort 2), with an overall accuracy of $79.1 \%$. In detail, high levels of expression of p-STAT3 were significantly associated with a high risk of disease (adjusted OR: 13.8, 95\% Cl: 4.3-44.3), and the final score was

$$
\operatorname{logit}(p)=-0.3254+2.628 \times p-S T A T 3(\text { GEO MFI }) \text {. }
$$


When glioma patients were considered according to their grade, intensity of p-STAT3 was confirmed to be an independent significant predictor of all grades of disease (Table 2a, accuracy of $68.6 \%$ ). From these results it thus appears that high levels of STAT3 activation in monocytes is not only a marker of immune suppression, but also a biomarker of disease.

The same analysis performed with soluble biomarkers identified ARG1 activity at physiological pH (Urea pH 7.2) as an independent risk factor of disease (adjusted OR: $255,95 \% \mathrm{Cl}: 26.7-2434$ ), with an overall accuracy of $94 \%$ and $\operatorname{git}(p)=-1.447+5.541 \times$ Urea $p H 7.2$.

When this analysis considered glioma patients according to their grade, ARG1 activity at pH.7.2 remained an independent significant predictor of all grades of disease, and, interestingly, significantly discriminated $\mathrm{HD}$ from low-grade gliomas, thus indicating that it is an early biomarker of glioma disease (Table $2 \mathrm{~b}$, cohort 3), with an accuracy of $72 \%$.

\section{Development of a GBM prognostic model}

We next evaluated the prognostic role of the myeloid-associated biomarkers present in this study to predict the outcome of GBM patients by performing a univariate analysis (Table S4), and then using multiple survival analyses (Table 3). Both analyses examined a cohort of 67 patients for myeloid cell markers (cohort 1), of 45 patients for PD-L1 and p-STAT3 (cohort 2), and of 32 patients for ARG1 markers (cohort 3) with available clinical and follow-up data (Table S5). At an estimated median follow-up time of 33.2 months $(95 \% \mathrm{Cl} 30.5-49.1)$ for cohort 1, 27.6 months (95\% Cl 19.3-30.5) for cohort 2, and 33.2 months (95\% Cl 28.9-37.7) for cohort 3, median OS times were 12.6 months (95\% Cl 10.6-18), 12.5 months (95\% $\mathrm{Cl} 6.2-16.7)$, and 12.0 months (95\% Cl 6.5-28.5), respectively.

In the multiple Cox regression model for the myeloid cell-associated markers, elevated MDSC2 levels remained significantly associated with worse OS (hazard ratio $(\mathrm{HR})=1.8,95 \% \mathrm{Cl}: 1.0-3.4$ ), in addition to ECOG PS, surgery and MGMT methylation (Table 3; C-index=0.74). The prognostic index derived from the model was:

\section{Prognostic risk score $=1.017 \times P S-0.935 \times$ Surgery $-0.689 \times M G M T+0.607 \times M D S C 2$}

Among STAT3 and PDL-1 markers, elevated levels of expression of p-STAT3 were significantly associated with worse overall survival $(\mathrm{HR}=4.43,95 \% \mathrm{Cl}$ : 1.7-11.6), in addition to surgery, Stupp and MGMT methylation (Table 3; C-index=0.75),

Prognostic risk score $=-2.323 \times$ Surgery $-1.551 \times$ Stupp $-1.691 \times$ MGMT $+1.488 \times p$-STAT3 $($ GEO MFI $)$.

The multiple prognostic model for ARG1 identified high levels of ARG1 activity at alkaline pH 9.5 as a risk factor for survival (HR=3.7, 95\% Cl: 1.4-9.9), in addition to Stupp treatment (Table 3; C-index=0.76),

Prognostic risk score $=-1.838 \times$ Stupp $+1.317 \times$ Urea $p H 9.5$. 


\section{Discussion}

In the present study, we evaluated the role of a number of factors that have been previously associated with immune suppressive activity due to myeloid cells in different tumor types. For example, in several studies a higher pretreatment MDSC level was significantly associated with worse OS $(4,39)$. Our study evaluated 4 subsets of MDCSs, from low to high-grade gliomas and found that 3 out of the 4 subsets are significantly increased in patients with increasing disease stage, thus reinforcing the notion that MDSCs are associated with disease progression. In addition, we found that levels of M-MDSC1 are also an independent factor significantly associated with a high risk of disease in a case-control study, and that it remained an independent factor discriminating different pathological stages of gliomas (Table 2). Thus, determination of MDSC1 levels represents a valuable tool associated with glioma diagnosis.

Determination of this parameter was carried out by multiparametric flow cytometry and we previously showed that monitoring MDSC subsets poses the problem of a lack of harmonization across different laboratories, and that standardization of common parameters is a necessary step to obtain intra and inter laboratory's reproducible results (8). Accordingly, for this study we standardized reagents and instrument for all the samples acquired, to guarantee a reliable comparison of the data across an extended period of time. As far as it concerns the prognostic role of MDSCs, we found that another subset of MDSC was significantly associated with outcome, since high levels of PMN-MDSC2 before surgical resection were associated to worst OS. On the other side, immature e-MDSC3 levels were significantly reduced in GII and GBM, as observed in a previous study with melanoma patients (7), and a prognostic independent risk factor for glioma versus HDs, and for discriminating different glioma pathological stages (Table 2). Reduction of this immature myeloid subset could be sustained by a dynamic but altered process of myeloid differentiation in cancer patients, giving rise to more differentiated myeloid cells toward monocytic or granulocytic MDSCs that, in fact, are increased in these patients. In line with this hypothesis, MDSC3 could represent a pool of circulating immature cells that gives rise to other MDSC subsets. Blood MDSC determination is an interesting option in the frame of a liquid biopsy, while tracking MDSCs in the tumor specimen is a challenging task, since such cells are virtually indistinguishable from tissue macrophages as they share myeloid markers and the functional immune suppressive activity. In addition, it has been demonstrated that MDSC differentiate to tumor-associated macrophages in the tumor microenvironment $(40,41)$.

Another finding that highlights an altered myelopoiesis in GBM patients is the increased presence of monocytes and PMNs, beside MDSC1, 2 and 4 (Fig. 2). Accordingly, expanded monocytes bear markers of immune suppression such as p-STAT3, and of immune dysfunction, like PD-L1, highlighting their involvement in the immune derangement process in gliomas. Furthermore, intensity of p-STAT3 in monocytes is another independent prognostic factor capable to discriminate gliomas versus HDs. As previously reported, STAT3 regulates ARG1 in MDSCs from cancer patients (21), and in our study we found that its quantity and activity are significantly increased in the blood of glioma patients. In addition, its activity at $\mathrm{pH} 7.2$ is another independent prognostic factor with diagnostic potential, but its activity at $\mathrm{pH} 9.5$ is prognostic factor for survival, independent of the treatment. Future studies will have to address the source and the role of this enzyme in the blood of glioma patients. In particular, secreted ARG1 is 
active as a full length protein at alkaline $\mathrm{pH}$, while it is inactive at neutral $\mathrm{pH}$ unless cleaved by PMNderived proteases (42). Thus, it will be interesting to understand whether ARG1 is actively secreted from the granules of PMNs, or released from immune suppressive monocytes, or M-MDSC, and whether an activation step is required to fully activate its potential. In this respect, recent results from our laboratories indicate that this enzyme has a complex and important role in immune suppression in pancreatic ductal adenocarcinoma and that exploitation of this pathway may enhance cancer immunotherapy (Canè, submitted manuscript), thus raising the possibility to exploit its use also in GBM.

All the results presented in this work require a validation in future independent studies. Of note, arginase activity at neutral $\mathrm{pH}$ and intensity of activated STAT3 hold potential to discriminate between the different glioma grading, but could also be used to monitor in glioma patients transition from low- to high-grade. Finally, monitoring the level of these biomarkers during treatment could be useful to link their changes with clinical outcome, especially in the context of new immunotherapeutic approaches. To date, immune-checkpoint inhibitors did not improve survival in glioma patients $(43,44)$. The identification of new prognostic factors like STAT3 and ARG1 might represent a critical step toward the development of new successful strategies of intervention in GBM. In fact, drugs targeting these molecular factors could be associated to immune-checkpoint inhibitor in order to increase their efficacy in glioma patients.

\section{Conclusion}

Results from this study indicate that dysfunctional myeloid cells and soluble factors in glioma patients may be not only a potential source of circulating biomarkers associated with disease stage and clinical outcome, but also highlight the altered interplay between immune system and tumor. In addition, these results indicate that simple blood tests may be used for glioma patient's stratification and management.

\section{Abbreviations}

ARG1: arginase-1

Cl: confidence interval

ECOG PS: ECOG performance status

FCM: flow cytometry

FMO: fluorescence minus one

GBM: glioblastoma

HD: healthy donors

HR: hazard ratio 
MDSC: myeloid derived suppressor cells

MFI: mean fluorescence intensity

OR: odds ratios

OS: overall survival

PBL: peripheral blood leukocytes

PBMC: peripheral blood mononuclear cells

PCA: principal component analysis

PD-L1: programmed death-ligand 1

PMN: polymorphonuclear cells

STAT3: Signal Transducer and Activator of Transcription 3

\section{Declarations}

\section{Ethics approval and consent to participate}

The study protocol was reviewed and approved by the ethical committee of the IOV-IRCCS and of Padova University Hospital (CESC IOV 2016/13 and 3848/AO/16, respectively) and all patients gave their informed written consent.

\section{Consent for publication}

Not applicable.

\section{Availability of data and materials}

The datasets during and/or analysed during the current study available from the corresponding author on reasonable request.

\section{Competing interests}

The authors declare that they have no competing interests.

\section{Funding}

This work was supported by Università degli Studi di Padova (CPDA-144873 to S.M.), Ministero della Salute (RF-2019-12369251 to SM) and of IOV-IRCCS (BIOV19MANDR to SM).

\section{Authors' contributions}


Conception and design: SM, ADP, PDB. Development of methodology: PDB, LP, SM, SC, EM, DB. Data acquisition: LP, SM, SC, EM. Analysis and interpretation of data: all Authors. Manuscript writing: all Authors.

\section{Acknowledgements}

We thank Maria Giovanna Russo and Pierantonio Gallo for help with graphics.

\section{References}

1. Louis DN, Perry A, Reifenberger G, von Deimling A, Figarella-Branger D, Cavenee WK, et al. The 2016 World Health Organization Classification of Tumors of the Central Nervous System: a summary. Acta Neuropathol. 2016;131(6):803-20.

2. Grabowski MM, Sankey EW, Ryan KJ, Chongsathidkiet P, Lorrey SJ, Wilkinson DS, et al. Immune suppression in gliomas. Journal of neuro-oncology. 2020.

3. Ugel S, De Sanctis F, Mandruzzato S, Bronte V. Tumor-induced myeloid deviation: when myeloidderived suppressor cells meet tumor-associated macrophages. J Clin Invest. 2015;125(9):3365-76.

4. Solito S, Marigo I, Pinton L, Damuzzo V, Mandruzzato S, Bronte V. Myeloid-derived suppressor cell heterogeneity in human cancers. Ann N Y Acad Sci. 2014;1319:47-65.

5. Veglia F, Sanseviero E, Gabrilovich DI. Myeloid-derived suppressor cells in the era of increasing myeloid cell diversity. Nat Rev Immunol. 2021.

6. Bronte V, Brandau S, Chen SH, Colombo MP, Frey AB, Greten TF, et al. Recommendations for myeloidderived suppressor cell nomenclature and characterization standards. Nature communications. 2016;7:12150.

7. Damuzzo V, Solito S, Pinton L, Carrozzo E, Valpione S, Pigozzo J, et al. Clinical implication of tumorassociated and immunological parameters in melanoma patients treated with ipilimumab. Oncoimmunology. 2016;5(12):e1249559.

8. Mandruzzato S, Brandau S, Britten CM, Bronte V, Damuzzo V, Gouttefangeas C, et al. Toward harmonized phenotyping of human myeloid-derived suppressor cells by flow cytometry: results from an interim study. Cancer Immunol Immunother. 2016;65(2):161-9.

9. Diaz-Montero CM, Salem ML, Nishimura MI, Garrett-Mayer E, Cole DJ, Montero AJ. Increased circulating myeloid-derived suppressor cells correlate with clinical cancer stage, metastatic tumor burden, and doxorubicin-cyclophosphamide chemotherapy. Cancer Immunol Immunother. 2009;58(1):49-59.

10. Solito S, Falisi E, Diaz-Montero CM, Doni A, Pinton L, Rosato A, et al. A human promyelocytic-like population is responsible for the immune suppression mediated by myeloid-derived suppressor cells. Blood. 2011;118(8):2254-65.

11. Walter S, Weinschenk T, Stenzl A, Zdrojowy R, Pluzanska A, Szczylik C, et al. Multipeptide immune response to cancer vaccine IMA901 after single-dose cyclophosphamide associates with longer 
patient survival. Nat Med. 2012.

12. Gielen PR, Schulte BM, Kers-Rebel ED, Verrijp K, Bossman SA, Ter Laan M, et al. Elevated levels of polymorphonuclear myeloid-derived suppressor cells in patients with glioblastoma highly express S100A8/9 and arginase and suppress T cell function. Neuro Oncol. 2016;18(9):1253-64.

13. Gielen PR, Schulte BM, Kers-Rebel ED, Verrijp K, Petersen-Baltussen HM, ter Laan M, et al. Increase in both CD14-positive and CD15-positive myeloid-derived suppressor cell subpopulations in the blood of patients with glioma but predominance of CD15-positive myeloid-derived suppressor cells in glioma tissue. J Neuropathol Exp Neurol. 2015;74(5):390-400.

14. Otvos B, Silver DJ, Mulkearns-Hubert EE, Alvarado AG, Turaga SM, Sorensen MD, et al. Cancer Stem Cell-Secreted Macrophage Migration Inhibitory Factor Stimulates Myeloid Derived Suppressor Cell Function and Facilitates Glioblastoma Immune Evasion. Stem Cells. 2016;34(8):2026-39.

15. Raychaudhuri B, Rayman P, Ireland J, Ko J, Rini B, Borden EC, et al. Myeloid-derived suppressor cell accumulation and function in patients with newly diagnosed glioblastoma. Neuro Oncol. 2011;13(6):591-9.

16. Soler DC, Young AB, Cooper KD, Kerstetter-Fogle A, Barnholtz-Sloan JS, Gittleman H, et al. The ratio of HLA-DR and VNN2(+) expression on CD14(+) myeloid derived suppressor cells can distinguish glioblastoma from radiation necrosis patients. Journal of neuro-oncology. 2017;134(1):189-96.

17. Alban TJ, Alvarado AG, Sorensen MD, Bayik D, Volovetz J, Serbinowski E, et al. Global immune fingerprinting in glioblastoma patient peripheral blood reveals immune-suppression signatures associated with prognosis. JCl insight. 2018;3(21).

18. Peereboom DM, Alban TJ, Grabowski MM, Alvarado AG, Otvos B, Bayik D, et al. Metronomic capecitabine as an immune modulator in glioblastoma patients reduces myeloid-derived suppressor cells. JCl insight. 2019;4(22).

19. Gabrilovich DI. Myeloid-Derived Suppressor Cells. Cancer immunology research. 2017;5(1):3-8.

20. Trovato R, Fiore A, Sartori S, Cane S, Giugno R, Cascione L, et al. Immunosuppression by monocytic myeloid-derived suppressor cells in patients with pancreatic ductal carcinoma is orchestrated by STAT3. J immunother Cancer. 2019;7(1):255.

21. Vasquez-Dunddel D, Pan F, Zeng Q, Gorbounov M, Albesiano E, Fu J, et al. STAT3 regulates arginaseI in myeloid-derived suppressor cells from cancer patients. J Clin Invest. 2013;123(4):1580-9.

22. Lemos H, Huang L, Prendergast GC, Mellor AL. Immune control by amino acid catabolism during tumorigenesis and therapy. Nature reviews. 2019;19(3):162-75.

23. Pham TN, Liagre B, Girard-Thernier C, Demougeot C. Research of novel anticancer agents targeting arginase inhibition. Drug discovery today. 2018;23(4):871-8.

24. Sippel TR, White J, Nag K, Tsvankin V, Klaassen M, Kleinschmidt-DeMasters BK, et al. Neutrophil degranulation and immunosuppression in patients with GBM: restoration of cellular immune function by targeting arginase I. Clin Cancer Res. 2011;17(22):6992-7002.

25. Lamano JB, Lamano JB, Li YD, DiDomenico JD, Choy W, Veliceasa D, et al. Glioblastoma-Derived IL6 Induces Immunosuppressive Peripheral Myeloid Cell PD-L1 and Promotes Tumor Growth. Clin Cancer 
Res. 2019;25(12):3643-57.

26. Peranzoni E, Ingangi V, Masetto E, Pinton L, Marigo I. Myeloid Cells as Clinical Biomarkers for Immune Checkpoint Blockade. Frontiers in immunology. 2020;11:1590.

27. Westphal M, Lamszus K. Circulating biomarkers for gliomas. Nat Rev Neurol. 2015;11(10):556-66.

28. Mandruzzato S, Solito S, Falisi E, Francescato S, Chiarion-Sileni V, Mocellin S, et al. IL4Ralpha + myeloid-derived suppressor cell expansion in cancer patients. J Immunol. 2009;182(10):6562-8.

29. Perfetto SP, Ambrozak D, Nguyen R, Chattopadhyay P, Roederer M. Quality assurance for polychromatic flow cytometry. Nature protocols. 2006;1(3):1522-30.

30. Hand DJ. Screening vs Prevalence Estimation. Journal of the Royal Statistical Society Series C (Applied Statistics). 1987;36(1):1-7.

31. Gabrusiewicz K, Rodriguez B, Wei J, Hashimoto Y, Healy LM, Maiti SN, et al. Glioblastoma-infiltrated innate immune cells resemble M0 macrophage phenotype. JCl insight. 2016;1(2).

32. Harshyne LA, Nasca BJ, Kenyon LC, Andrews DW, Hooper DC. Serum exosomes and cytokines promote a T-helper cell type 2 environment in the peripheral blood of glioblastoma patients. Neuro Oncol. 2016;18(2):206-15.

33. Pinton L, Masetto E, Vettore M, Solito S, Magri S, D'Andolfi M, et al. The immune suppressive microenvironment of human gliomas depends on the accumulation of bone marrow-derived macrophages in the center of the lesion. J immunother Cancer. 2019;7(1):58.

34. Abramson N, Melton B. Leukocytosis: basics of clinical assessment. Am Fam Physician. 2000;62(9):2053-60.

35. Dubinski D, Won SY, Gessler F, Quick-Weller J, Behmanesh B, Bernatz S, et al. Dexamethasoneinduced leukocytosis is associated with poor survival in newly diagnosed glioblastoma. Journal of neuro-oncology. 2018;137(3):503-10.

36. Moyes KW, Davis A, Hoglund V, Haberthur K, Lieberman NA, Kreuser SA, et al. Effects of tumor grade and dexamethasone on myeloid cells in patients with glioma. Oncoimmunology. 2018;7(11):e1507668.

37. Damuzzo V, Pinton L, Desantis G, Solito S, Marigo I, Bronte V, et al. Complexity and challenges in defining myeloid-derived suppressor cells. Cytometry B Clin Cytom. 2015;88(2):77-91.

38. Bazhin AV, von Ahn K, Fritz J, Werner J, Karakhanova S. Interferon-alpha Up-Regulates the Expression of PD-L1 Molecules on Immune Cells Through STAT3 and p38 Signaling. Frontiers in immunology. 2018;9:2129.

39. Wang PF, Song SY, Wang TJ, Ji WJ, Li SW, Liu N, et al. Prognostic role of pretreatment circulating MDSCs in patients with solid malignancies: A meta-analysis of 40 studies. Oncoimmunology. 2018;7(10):e1494113.

40. Kumar V, Cheng P, Condamine T, Mony S, Languino LR, McCaffrey JC, et al. CD45 Phosphatase Inhibits STAT3 Transcription Factor Activity in Myeloid Cells and Promotes Tumor-Associated Macrophage Differentiation. Immunity. 2016;44(2):303-15. 
41. Kwak T, Wang F, Deng H, Condamine T, Kumar V, Perego M, et al. Distinct Populations of ImmuneSuppressive Macrophages Differentiate from Monocytic Myeloid-Derived Suppressor Cells in Cancer. Cell reports. 2020;33(13):108571.

42. Rotondo R, Bertolotto M, Barisione G, Astigiano S, Mandruzzato S, Ottonello L, et al. Exocytosis of azurophil and arginase 1-containing granules by activated polymorphonuclear neutrophils is required to inhibit T lymphocyte proliferation. J Leukoc Biol. 2011;89(5):721-7.

43. Lombardi G, Barresi V, Indraccolo S, Simbolo M, Fassan M, Mandruzzato S, et al. Pembrolizumab Activity in Recurrent High-Grade Gliomas with Partial or Complete Loss of Mismatch Repair Protein Expression: A Monocentric, Observational and Prospective Pilot Study. Cancers. 2020;12(8).

44. Reardon DA, Brandes AA, Omuro A, Mulholland P, Lim M, Wick A, et al. Effect of Nivolumab vs Bevacizumab in Patients With Recurrent Glioblastoma: The CheckMate 143 Phase 3 Randomized Clinical Trial. JAMA Oncol. 2020;6(7):1003-10.

\section{Tables}

Table 2a. Factors for glioma risk (multiple analysis with logistic regression model).

\begin{tabular}{|lllll|}
\hline & & E/N & Adjusted OR (95\%Cl) & P(LR-test) \\
\hline Cohort 1 & & & & \\
\hline CD 15 ${ }^{+}$ & Low & $16 / 39$ & Ref & \\
& High & $88 / 101$ & $7.2(2.2-24.1)$ & $<0.001$ \\
\hline \% MDSC1 & Low & $12 / 40$ & Ref & \\
\hline Cohort 2 & High & $92 / 100$ & $40.1(10.0-160.4)$ & $<0.001$ \\
\hline p-STAT3 (geo MFI) & Low & $89 / 105$ & Ref & \\
\hline & High & $15 / 35$ & $0.1(0.02-0.4)$ & $<0.001$ \\
\hline Cohort 3 & High & $50 / 55$ & $13.8(4.3-44.3)$ & $<0.001$ \\
\hline Urea pH 7.2 & & & & \\
\hline & Low & $4 / 21$ & Ref & \\
\hline
\end{tabular}

OR, odds ratio; $95 \% \mathrm{Cl}, 95 \%$ confidence interval; $\mathrm{E}$, number of glioma patients; $\mathrm{N}$, total number of subjects; LR, Likelihood ratio. 
Table 2b. Factors for glioma grade risk (multiple analysis with multinomial logistic regression model).

\begin{tabular}{|c|c|c|c|c|}
\hline & & Adjusted OR (95\% Cl) & $\begin{array}{l}\text { Adjusted OR (95\% } \\
\text { Cl) }\end{array}$ & Adjusted OR (95\% Cl) \\
\hline & & GBM & GII & GIII \\
\hline \multicolumn{5}{|l|}{ Cohort 1} \\
\hline Age & Cont. & $1.01(0.96-1.07)$ & $0.9(0.85-0.96)^{\star \star}$ & $0.95(0.9-1.01)$ \\
\hline \multirow[t]{2}{*}{$\% \mathrm{CD} 15^{+}$} & Low & Ref & Ref & Ref \\
\hline & High & $32.6(6.3-168.3)^{\star \star \star}$ & $2.8(0.5-14.7)$ & $2.9(0.6-15.2)$ \\
\hline \multirow[t]{2}{*}{$\%$ MDSC1 } & Low & Ref & Ref & Ref \\
\hline & High & $65.0(12.5-338.2)^{\star \star \star}$ & $28.8(4.3-193.7)^{\star \star \star}$ & $28.0(4.0-194.7)^{\star \star \star}$ \\
\hline \multirow[t]{2}{*}{$\%$ MDSC3 } & Low & Ref & Ref & Ref \\
\hline & High & $0.07(0.01-0.35)^{\star *}$ & $0.06(0.01-0.44)^{\star \star}$ & $0.19(0.03-1.11)$ \\
\hline \multicolumn{5}{|l|}{ Cohort 2} \\
\hline Age & Cont. & $1.06(1.0-1.13)^{\star}$ & $0.92(0.85-1)$ & $0.98(0.91-1.07)$ \\
\hline \multirow{2}{*}{$\begin{array}{l}\text { p-STAT3 (geo } \\
\text { MFI) }\end{array}$} & Low & Ref & Ref & Ref \\
\hline & High & $11.2(3.3-38.6)^{\star \star \star}$ & $17.3(1.6-192)^{\star}$ & $17.6(1.65-188.5)^{\star}$ \\
\hline \multicolumn{5}{|l|}{ Cohort 3} \\
\hline Age & Cont. & $1.18(1.06-1.32)^{\star \star}$ & $1.04(0.94-1.15)$ & $1.09(0.98-1.21)$ \\
\hline \multirow[t]{2}{*}{ Urea $\mathrm{pH} 7.2$} & Low & Ref & Ref & Ref \\
\hline & High & $\begin{array}{l}8382 \\
(124.4-564647)^{\star \star \star}\end{array}$ & $\begin{array}{l}433.5 \\
(12.6-14858)^{\star \star \star}\end{array}$ & $\begin{array}{l}291.8 \\
(7.74-11009.3)^{\star *}\end{array}$ \\
\hline
\end{tabular}

Significance levels: $<0.001: * * * ;<0.01: * * ;<0.05:$ *

Table 3. Factors for GBM patients' survival (multivariate analysis with Cox proportional hazards model). 


\begin{tabular}{|c|c|c|c|c|c|}
\hline & & $E / N$ & median (95\% Cl) & Adjusted HR (95\% Cl) & $\mathrm{P}$ (LR-test) \\
\hline \multicolumn{6}{|l|}{ Cohort 1} \\
\hline \multirow[t]{2}{*}{ ECOG PS } & $0-1$ & $32 / 41$ & $18.3(14.0,22.1)$ & Ref & \\
\hline & $2-3$ & $24 / 26$ & $6.0(4.2,10.6)$ & $2.8(1.5,4.9)$ & $<0.001$ \\
\hline \multirow[t]{2}{*}{ Surgery } & Other & $47 / 53$ & $11.2(6.4,14.4)$ & Ref & \\
\hline & Radical & $9 / 14$ & $25.9(12.0, \mathrm{NE})$ & $0.4(0.2,0.8)$ & 0.009 \\
\hline MGMT promoter & No & $28 / 30$ & $11.3(6.5,13.0)$ & Ref & \\
\hline methylation & Yes & $28 / 37$ & $18.0(10.8,22.8)$ & $0.5(0.3,0.9)$ & 0.015 \\
\hline \multirow[t]{2}{*}{ \% MDSC2 } & Low & $14 / 20$ & $21.4(5.9,33.0)$ & Ref & \\
\hline & High & $42 / 47$ & $11.5(7.7,14.4)$ & $1.8(1.0,3.4)$ & 0.047 \\
\hline \multicolumn{6}{|l|}{ Cohort 2} \\
\hline \multirow[t]{2}{*}{ Surgery } & Other & $31 / 39$ & $10.6(5.9,16.0)$ & Ref & \\
\hline & Radical & $1 / 6$ & - & $0.1(0.01,0.8)$ & 0.002 \\
\hline \multirow[t]{2}{*}{ Stupp's regimen } & No & $8 / 9$ & $2.7(1.8,8.2)$ & Ref & \\
\hline & Yes & $24 / 36$ & $15.5(9.2,22.8)$ & $0.2(0.09,0.5)$ & 0.002 \\
\hline MGMT promoter & No & $16 / 18$ & $8.5(3.1,13.0)$ & Ref & \\
\hline methylation & Yes & $16 / 27$ & $21.6(6.2, \mathrm{NE})$ & $0.2(0.1,0.5)$ & $<0.001$ \\
\hline \multirow[t]{2}{*}{ p-STAT3 (geo MFI) } & Low & $22 / 30$ & $15.4(8.7,21.6)$ & Ref & \\
\hline & High & $10 / 15$ & $6.2(2.7, \mathrm{NE})$ & $4.4(1.7,11.6)$ & 0.003 \\
\hline \multicolumn{6}{|l|}{ Cohort 3} \\
\hline \multirow[t]{2}{*}{ Stupp's regimen } & No & $5 / 5$ & 2.3 (0.6-NE) & Ref & \\
\hline & Yes & $18 / 27$ & $20.0(8.2-33.0)$ & $0.2(0.05,0.5)$ & 0.005 \\
\hline \multirow[t]{2}{*}{ Urea pH 9.5} & Low & $7 / 16$ & - & Ref & \\
\hline & High & $16 / 16$ & $8.0(5.7-13.0)$ & $3.7(1.4,9.9)$ & 0.007 \\
\hline
\end{tabular}

$\mathrm{HR}$, hazard ratio; $95 \% \mathrm{Cl}, 95 \%$ confidence interval; $\mathrm{E}$, number of deaths; $\mathrm{N}$, total number of glioblastoma patients; NE, not estimable; LR, Likelihood ratio.

Due to technical limitations, Table 1 is only available as a download in the Supplemental Files section. 


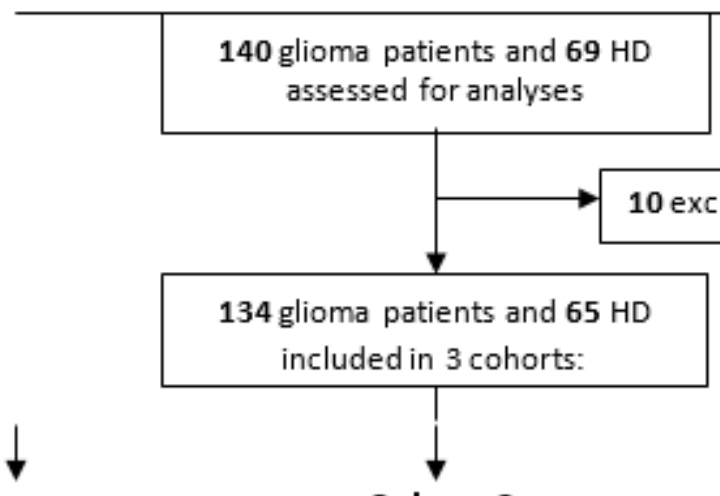

Cohort 1

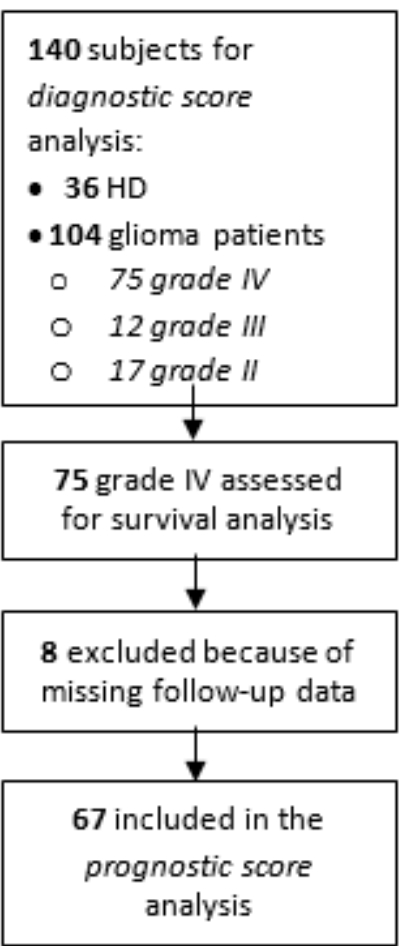

Cohort 2

86 subjects for diagnostic score analysis:

- $23 \mathrm{HD}$

- 63 glioma patients

- 49 grade $I V$

- 6 grade III

- 8 grade II

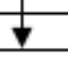

49 grade IV assessed for survival analysis

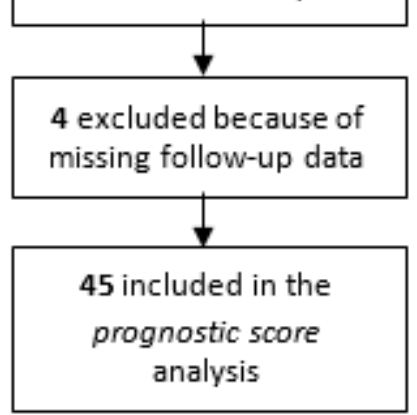

$\mathbf{8 2}$ subjects for diagnostic score analysis:

- $18 \mathrm{HD}$

- 64 glioma patients

- 39 grade IV

- 10 grade III

- 15 grade II

39 grade IV assessed for survival analysis

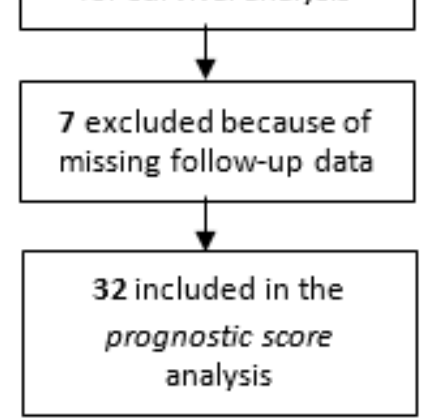

\section{Figure 1}

\section{Consort Statement}



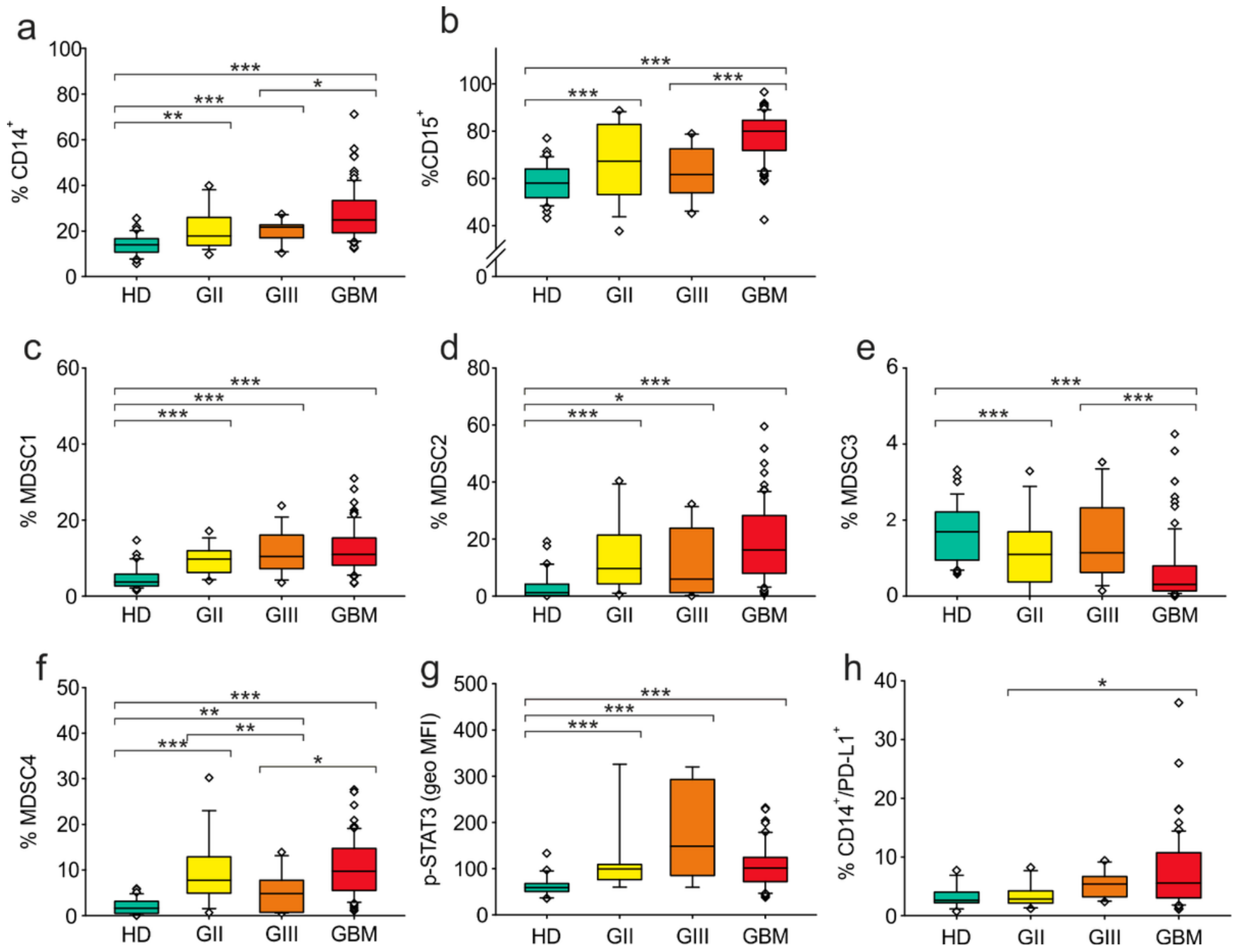

Figure 2

Distribution of circulating myeloid cells in glioma patients. Pairwise comparison of cell-associated markers between HDs and low- and high-grade glioma patients were performed using a two-tailed MannWhitney test, adjusted for sex and age followed by the Benjamini-Hochberg multiple testing correction. Box Plots show the median, 25th and 75th percentile of the percentage of (a) monocytes (CD14+ cells among PBMCs) (36 HDs, 18 grade II gliomas, 13 grade III gliomas and 78 GBM), (b) granulocytes, (CD15+ cells among peripheral blood leukocytes, PBLs) (36 HDs, 18 grade II gliomas, 13 grade III gliomas and 79 GBM), (c) MDSC1, (CD14+/IL4Ra+ cells among PBMCs) (36 HDs, 18 grade II gliomas, 13 grade III gliomas and 79 GBM), (d) MDSC2 (CD15+/IL4Ra+ cells among PMNs) (36 HDs, 17 grade II gliomas, 12 grade III gliomas and 79 GBM), (e) MDSC3 (Lin-/HLA-DR-/CD11b+/CD33+ cells) in CD15-cells (36 HDs, 18 grade II gliomas, 13 grade III gliomas and 79 GBM), (f) MDSC4 (CD14+/HLA-DRlow/- cells among PBMCs) (36 HDs, 18 grade II gliomas, 13 grade III gliomas and $76 \mathrm{GBM}$ ). Whiskers extend to 1.5 interquartile range and outliers are shown by dots. (g) The geometric mean fluorescence intensity of p-STAT3 (p-STAT3, geo MFI) expression compared to a negative control was evaluated by flow cytometry in CD14+ 
cells among PBMCs of 23 HDs and glioma patients (8 grade II, 6 grade III and 50 GBM), following intracellular staining. (h) Surface expression of PD-L1 was evaluated by flow cytometry in the peripheral blood of HDs and glioma patients, by gating CD14+/PD-L1+ cells among PBMCs (29 HDs, 14 grade II gliomas, 10 grade III gliomas and 61 GBM). Only statistically significant comparisons are reported in figure: $<0.001: * * * ;<0.01: * * ;<0.05: *$.
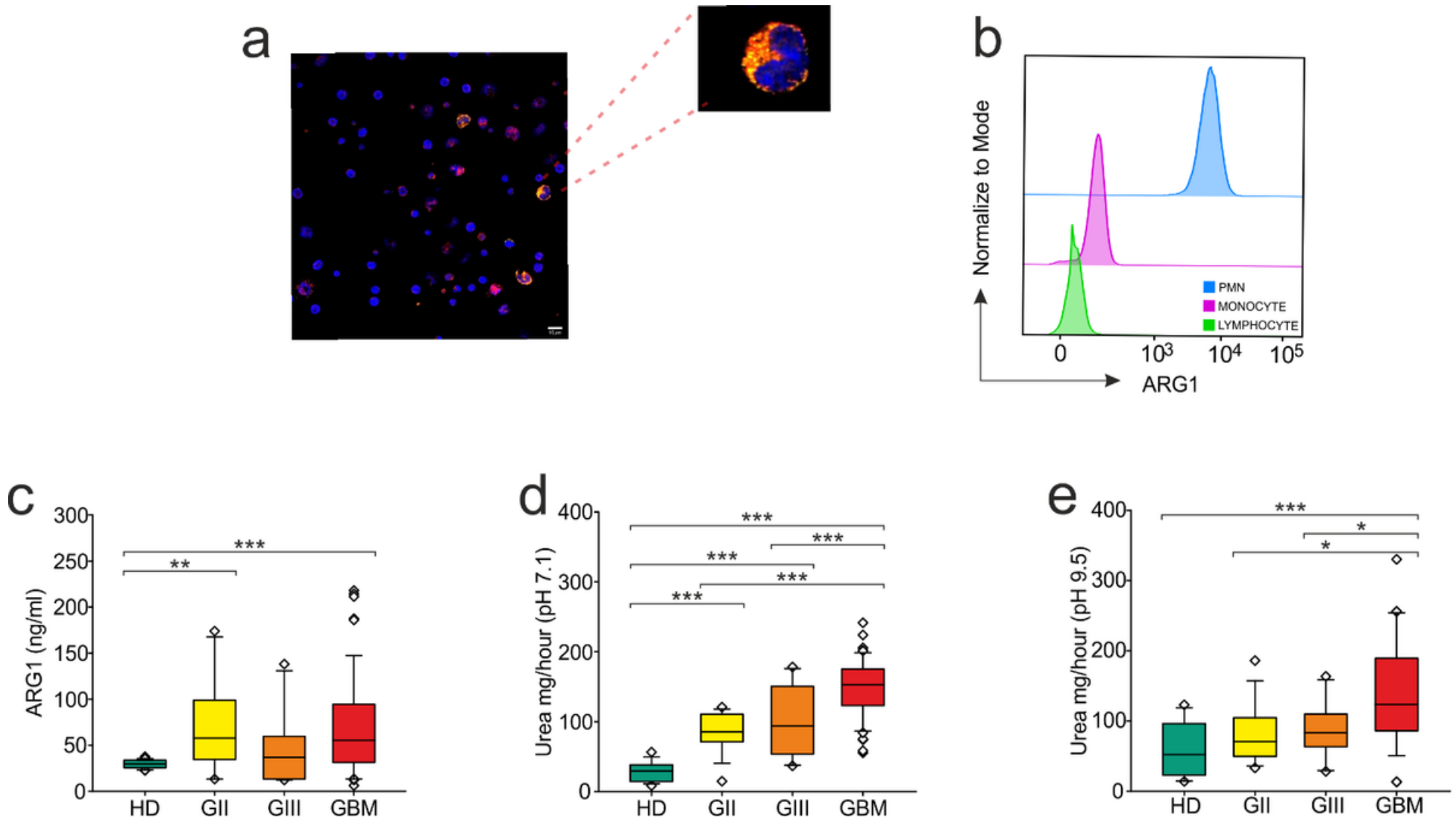

Figure 3

Evaluation of blood ARG1 presence and activity. (a) Representative image of confocal microscopy analysis performed on PBMCs from glioma patients. ARG1+ cells are shown in yellow and CD14+ cells in red. Slides were analyzed at a $63 \mathrm{X}$ magnification and cell size is reported by scale bar (10 $\mu \mathrm{m})$. Upper right panel shows a 189X magnification of a monocyte positive for both ARG1 and CD14 markers from a GBM patient. (b) Flow cytometry analysis of ARG1 in PMNs (blue), monocytes (pink) and lymphocytes (green). (c) Levels of ARG1 evaluated by ELISA (22 HDs, 15 grade II gliomas, 10 grade III gliomas and 57 GBM). ARG1 functional activity was tested by urea assay in the plasma of HDs and glioma patients at a $7.2 \mathrm{pH}$ (18 HDs, 15 grade II gliomas, 10 grade III gliomas and 57 GBM) (d) and at pH 9.5 pH (18 HDs, 15 grade II gliomas, 10 grade III gliomas and 39 GBM) (e). Pairwise comparison between HDs and low- and high-grade glioma patients were performed using a two-tailed Mann-Whitney test, adjusted for sex and age, followed by the Benjamini-Hochberg multiple testing correction. Only statistically significant comparisons are reported in figure: <0.001: ***; $<0.01: * \star ;<0.05:$. 


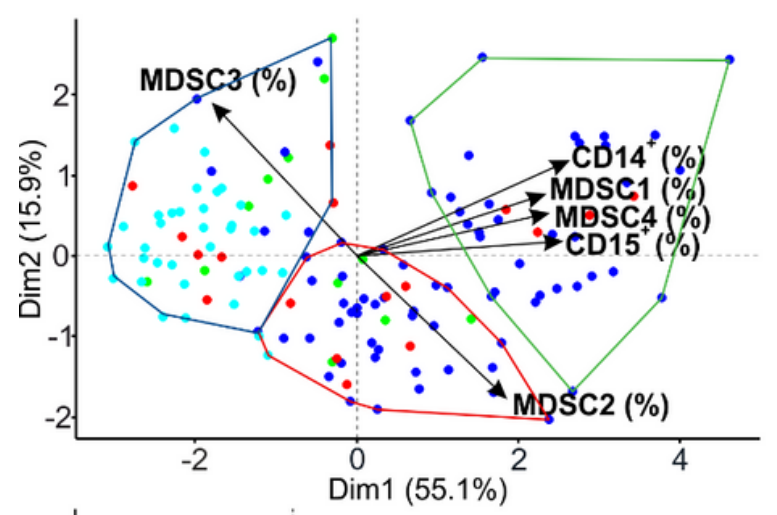

\begin{tabular}{lccccc} 
& HD & GII & GII & GBM $\bullet$ & RowTotal \\
\hline Cluster1 & 35 & 7 & 7 & 7 & 56 \\
& $62.5 \%$ & $12.5 \%$ & $12.5 \%$ & $12.5 \%$ & \\
& $97.2 \%$ & $41.2 \%$ & $58.3 \%$ & $9.3 \%$ & $40 \%$ \\
\hline Cluster2 & 1 & 6 & 5 & 36 & 48 \\
& $2.1 \%$ & $12.5 \%$ & $10.4 \%$ & $75 \%$ & \\
& $2.8 \%$ & $35.3 \%$ & $41.7 \%$ & $48 \%$ & $34.3 \%$ \\
\hline Cluster3 & 0 & 4 & 0 & 32 & 36 \\
& 0 & $11.1 \%$ & 0 & $88.9 \%$ & \\
& 0 & $23.5 \%$ & 0 & $42.7 \%$ & $25.7 \%$ \\
\hline Total & 36 & 17 & 12 & 75 & 140 \\
& $25.7 \%$ & $12.1 \%$ & $8.6 \%$ & $53.6 \%$ & \\
\hline
\end{tabular}

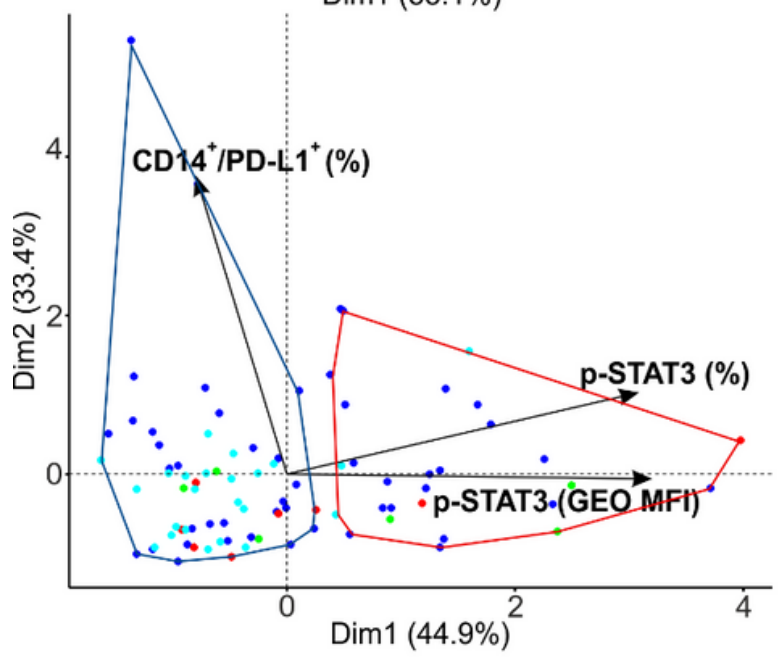

\begin{tabular}{lccccc} 
& HD & GII & GIII & GBM $\bullet$ & RowTotal \\
\hline Cluster1 & 20 & 6 & 3 & 29 & 58 \\
& $34.5 \%$ & $10.3 \%$ & $5.2 \%$ & $50 \%$ & \\
& $87 \%$ & $75 \%$ & $50 \%$ & $59.2 \%$ & $67.4 \%$ \\
\hline Cluster2 & 3 & 2 & 3 & 20 & 28 \\
& $10.7 \%$ & $7.1 \%$ & $10.7 \%$ & $71.4 \%$ & \\
& $13 \%$ & $25 \%$ & $50 \%$ & $40.8 \%$ & $32.6 \%$ \\
\hline Total & 23 & 8 & 6 & 49 & 86 \\
& $26.7 \%$ & $9.3 \%$ & $7 \%$ & $57 \%$ & \\
\hline
\end{tabular}

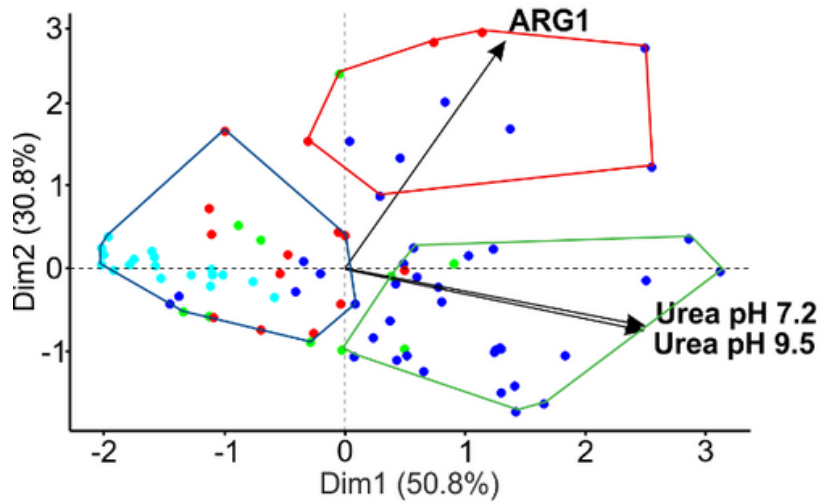

\begin{tabular}{lccccc} 
& HD & GII & GII & GBM & RowTotal \\
\hline Cluster1 & 18 & 11 & 5 & 6 & 40 \\
& $45 \%$ & $27.5 \%$ & $12.5 \%$ & $15 \%$ & \\
& $100 \%$ & $73.3 \%$ & $50 \%$ & $15.4 \%$ & $48.8 \%$ \\
\hline Cluster2 & 0 & 3 & 1 & 7 & 11 \\
& 0 & $27.3 \%$ & $9.1 \%$ & $63.6 \%$ & \\
& 0 & $20 \%$ & $10 \%$ & $17.9 \%$ & $13.4 \%$ \\
\hline Cluster3 & 0 & 1 & 4 & 26 & 31 \\
& 0 & $3.2 \%$ & $12.9 \%$ & $83.9 \%$ & \\
& 0 & $6.7 \%$ & $40 \%$ & $66.7 \%$ & $37.8 \%$ \\
\hline Total & 18 & 15 & 10 & 39 & 82 \\
& $22 \%$ & $18.3 \%$ & $12.2 \%$ & $47.6 \%$ & \\
\hline
\end{tabular}

Figure 4

PCA bidimensional plot of candidate biomarkers and cluster analysis. Distribution of glioma patients and HD divided by clusters on the right side. The numbers on the first line refers to the number of cases, in the second line the row percentage, the third line the column percentage.

\section{Supplementary Files}

This is a list of supplementary files associated with this preprint. Click to download.

- Table1.docx

- SupplementaryData.docx 